\title{
Covid-19: Doctors and nurses donate samples to study disease
}

\author{
Jacqui Wise \\ London
}

Doctors and nurses working at the new emergency Nightingale Hospital in east London will be studied as part of a new research project into covid-19, which aims to provide answers about the early stage of the disease and why it affects some people more than others.

The hospital's head of research, James Moon, is appealing for public donations to fund the project to avoid the long delays that can happen with conventional funding.

"We have no funding currently," he told The BMJ. "The pandemic is a fast evolving situation, and we took the decision that we had to start immediately and find funding later." $\mathrm{He}$ estimated that $£ 1 \mathrm{~m}(€ 1.14 \mathrm{~m} ; \$ 1.24 \mathrm{~m})$ would be needed to set up the core architecture of the project.

The research-a collaboration between Bart's Health, University College London, and Queen Mary University London—will build up a unique library of blood and swab samples from healthcare workers.

The research team, called the Covid Consortium, has already recruited 400 healthcare professionals from Bart's Hospital who have provided blood samples and data. The plan is to recruit a further 600 from the new Nightingale Hospital. Samples will be taken weekly for 16 weeks, and longer term follow-up is also planned at six and 12 months.

\section{High rates of exposure}

The biorepository will contain over 200000 blood and swab samples that will then be sent out to 25 of the UK's leading academic and pharmaceutical research institutions, which have developed distinct research questions that they hope to answer by using the data.

Most research into covid-19 is focusing on patients admitted to hospital with the disease, whereas studying healthcare workers who experience high rates of exposure to the virus will allow researchers to gather important information and samples from people before, during, and after they develop the disease.

"This project is about mild disease or those who are asymptomatic," Moon told The BMJ. "The samples will be critical to tell us about the genetics, immunity, presence of antibodies, and cell responses in these people."

He said that the consortium had "franchised" the study for researchers in Cape Town and Sydney. This will allow researchers in the southern hemisphere to answer other questions about covid-19 such as on ethnicity, vitamin D for immunity, and the changing seasons.

This is a global problem, and it is important to share research and resources, said Moon. "We are trying to pack what was achieved over a decade of HIV work into six months," he added.

\section{Key questions}

The research project has moved extremely swiftly: the idea was first discussed on 13 March, ethics and regulatory approval was gained on 19 March, and the first 400 healthcare workers were recruited on 1 April. The first analyses will be done as early as next week. Moon said that he hoped any findings would be shared as soon as possible but that each part of the study would be analysed separately.

Charlotte Manisty, consultant cardiologist at University College London and Barts Heart Centre, who is also working on the project, added, "It's a challenging time at the moment, and we obviously were very careful to make sure we did not take any resources or staff away from the frontline NHS."

The research will investigate the genetics of the response to the disease. Key questions on covid-19 that the study hopes to answer will also include:

- Immunity: If people have had covid-19, even mildly, are they protected from getting a bad dose in the future? If someone is immune to covid-19 how long does this last? If some people are resistant to further infection can their antibodies be used to protect other people?

- Transmission: Where are the "hotspots" for catching the infection in a hospital setting? Does the virus mutate as it passes from person to person? If so, how does that affect how immune people are to covid-19-namely, could a person catch a subsequent version of the virus despite already having been infected?

- Vaccination: What new ways are there to attack the virus, and what new targets for drugs?

- Testing: How can the NHS mass produce testing? Could production line techniques be used to do thousands of tests a day and make it easier to collect samples? Could patients perform their own tests rather than attending a centre and requiring a healthcare professional to test them?

Anyone wishing to support the study can donate at https://www. justgiving.com/fundraising/bartscovid19. 
